\title{
Dynamic collaboration without communication: Vision-based cable-suspended load transport with two quadrotors
}

\author{
Gassner, Michael ; Cieslewski, Titus ; Scaramuzza, Davide
}

\begin{abstract}
Transport of objects is a major application in robotics nowadays. While ground robots can carry heavy payloads for long distances, they are limited in rugged terrains. Aerial robots can deliver objects in arbitrary terrains; however they tend to be limited in payload. It has been previously shown that, for heavy payloads, it can be beneficial to carry them using multiple flying robots. In this paper, we propose a novel collaborative transport scheme, in which two quadrotors transport a cable-suspended payload at accelerations that exceed the capabilities of previous collaborative approaches, which make quasistatic assumptions. Furthermore, this is achieved completely without explicit communication between the collaborating robots, making our system robust to communication failures and making consensus on a common reference frame unnecessary. Instead, they only rely on visual and inertial cues obtained from on-board sensors. We implement and validate the proposed method on a real system.
\end{abstract}

DOI: https://doi.org/10.1109/icra.2017.7989609

Posted at the Zurich Open Repository and Archive, University of Zurich

ZORA URL: https://doi.org/10.5167/uzh-138919

Conference or Workshop Item

Published Version

Originally published at:

Gassner, Michael; Cieslewski, Titus; Scaramuzza, Davide (2017). Dynamic collaboration without communication: Vision-based cable-suspended load transport with two quadrotors. In: IEEE International Conference on Robotics and Automation, Singapore, 29 May 2017 - 3 June 2017, IEEE.

DOI: https://doi.org/10.1109/icra.2017.7989609 


\title{
Dynamic Collaboration without Communication: Vision-Based Cable-Suspended Load Transport with Two Quadrotors
}

\author{
Michael Gassner, Titus Cieslewski and Davide Scaramuzza
}

\begin{abstract}
Transport of objects is a major application in robotics nowadays. While ground robots can carry heavy payloads for long distances, they are limited in rugged terrains. Aerial robots can deliver objects in arbitrary terrains; however they tend to be limited in payload. It has been previously shown that, for heavy payloads, it can be beneficial to carry them using multiple flying robots. In this paper, we propose a novel collaborative transport scheme, in which two quadrotors transport a cable-suspended payload at accelerations that exceed the capabilities of previous collaborative approaches, which make quasi-static assumptions. Furthermore, this is achieved completely without explicit communication between the collaborating robots, making our system robust to communication failures and making consensus on a common reference frame unnecessary. Instead, they only rely on visual and inertial cues obtained from on-board sensors. We implement and validate the proposed method on a real system.
\end{abstract}

\section{INTRODUCTION}

A major application of autonomous robots is the transport of objects. Transporting objects in a known environment using ground robots has been applied in industry for several years now. A controlled environment can be equipped with rails, guides or markers which make navigation trivial. Recent advances in GPS technology allow similar ease of navigation in situations where GPS is available. We deal with object transport in an unknown, GPS-denied environment.

We employ flying robots, specifically quadrotors, which in contrast to ground robots can also be deployed in very cluttered environments, such as would be typically encountered in search and rescue scenarios.

While being deployable in a wide range of scenarios, quadrotors in general have a much more limited payload capacity than ground robots. If a payload is to be transported with a single quadrotor, the quadrotor thrust capacity needs to increase linearly with the weight of the payload. In turn, in order to provide a higher trust the size and weight of the quadrotor would need to increase in order to accommodate larger motors and a larger battery. With current technology, even a moderate payload of a couple of kilograms would require a very large quadrotor. Apart from being more dangerous and more expensive, it is generally known in aviation that rotorcraft can scale only up to a certain size [1]. Besides, smaller quadrotors are generally more agile [2].

It has previously been proposed to employ multiple rotorcrafts to carry a single large payload, both for manned [3] and

The authors are with the Robotics and Perception Group, University of Zurich, Switzerland-http://www.ifi.uzh.ch/en/rpg.html

This research was funded by the Mohamed Bin Zayed International Robotics Challenge (MBZIRC) and NCCR Robotics through the Swiss National Science Foundation.

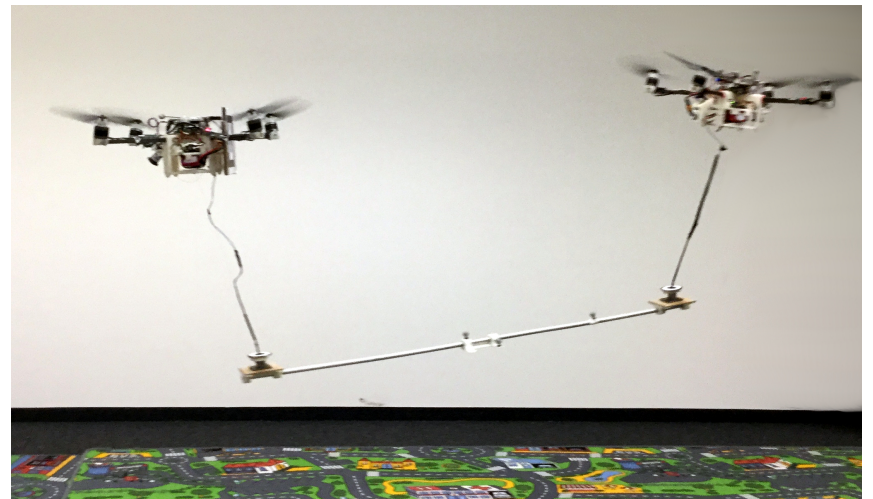

Fig. 1. We propose a system in which a cable-suspended load is collaboratively transported by two quadrotors at moderate speeds. This is achieved using on-board visual sensing only and without any explicit communication.

autonomous [4] rotorcraft. Apart from increasing the payload range of a multi-quadrotor system, carrying an object with multiple quadrotors makes it possible to control the orientation of the object without applying any torque to individual quadrotors [5]. Being able to control the orientation of the object can be useful to fit large objects through narrow gaps, or to minimize aerodynamic drag.

Orientation control can be achieved without applying torque given that the quadrotors attach to the object via cables. In fact, a well placed cable should allow each quadrotor to control its attitude without taking the payload into account. Furthermore, cables allow keeping the object at a distance, thus minimizing aerodynamic interference between the quadrotors and the load. A drawback of attaching to a mass via a cable is that this introduces additional mechanical dynamics. In its simplest form - a point mass tethered to a single quadrotor - this problem has recently been studied in [6], [7], [8], and we use their insights in the design of our controller. More complex tethered systems are among others modeled by [9], [10]. A system which uses arms instead of cables has been thus far only shown in simulation [11], [12].

In this paper, we propose a novel method for collaborative aerial transport of a cable-suspended object. Our method is distinct from previous work in the following two aspects: firstly, it achieves collaborative transport completely without explicit communication. This is achieved by heterogeneously distributing control over the degrees of freedom of the object, using a leader-follower approach [13]. The coordination cues that remain are inferred from visual observations of the transported object and the other quadrotors. Secondly, we model and control the dynamics of not just hovering, going 


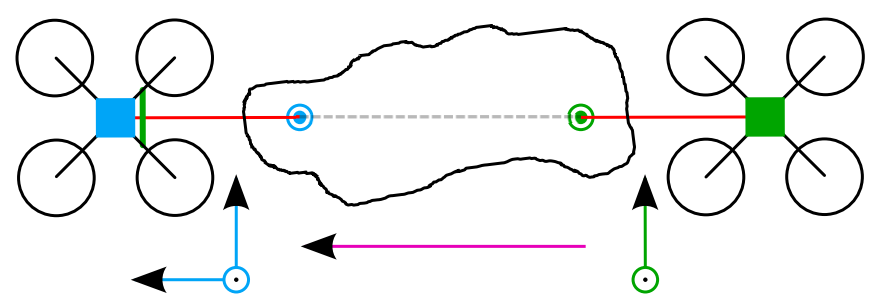

Fig. 2. Top view of our system. The leader (blue) controls the 3D position while the follower controls the transport direction (lilac) of the transported object. Each robot tracks the object using tags attached to the gripper. The follower tracks the leader using a tag attached to its back.

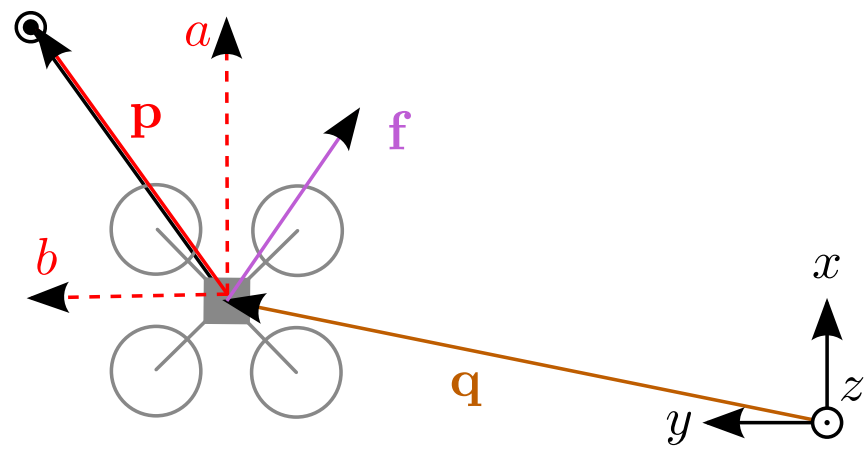

Fig. 3. Positions and forces in the simple slungload model. The pendulum position $\mathbf{p}$ is expressed with respect to the quadrotor position $\mathbf{q}$. We denote as $a$ and $b$ the $x$ and $y$ coefficients of $\mathbf{p}$. The force exerted by the quadrotor is denoted by $\mathbf{f}$.

beyond quasi-static object manipulation [5], [14], [15].

Not relying on explicit communication makes our system robust to communication outages and communication latency. This has previously been exploited in collaborative transport using ground robots [13], [16]. Moreover, no map sharing or consensus on a global reference frame is required, which is particularly desirable for applications where no global reference frame from GPS or from a motion capture system is available.

In our real world validation system, we rely solely on onboard sensing, using camera and IMU for quadrotor state estimation and control, and only a camera for observation and control of the mass and observation of the other robot. All computations are performed on-board the two quadrotors, and there is strictly zero communication during the execution of the transport task.

\section{Methodology}

Our system is composed of two quadrotors, a leader and a follower (see Fig. 2). In this section, we detail the control architecture of our leader-follower transportation system. The implementation details of the estimation are described in Section III.

To reduce the complexity and thus increase the robustness of the control architecture we design a control structure that remains stable with as little failure points as possible. We therefore choose to simplify the dual lift model [9] to two decoupled slung-load systems as shown in Fig. 3. This choice allows us to design a control scheme that relies solely on the gripper state estimate and the quadrotor state estimate, instead of having to estimate the full 6DOF state of the load to be transported as well as the 3DOF of the collaborative quadrotor state.

\section{A. Equation of Motion}

The equation of motion of the quadrotor with pendulum can be found in several papers [6], [7], [8]. We choose the parameterization proposed in [17] and apply it for the case of a normal instead of an inverted pendulum.

We model the quadrotor and the load as point masses with their positions parameterized as $\mathbf{q}=[x, y, z]^{T}$ and $\mathbf{p}=[a, b]^{T}$ respectively, as shown in Fig. 3. The position $\mathbf{p}_{\text {ext }}$ of the load in the world coordinate frame can be directly expressed from $\mathbf{q}$ and $\mathbf{p}$ as

$$
\mathbf{p}_{e x t}=\left[\begin{array}{c}
x+a \\
y+b \\
z-\sqrt{l^{2}-\left(a^{2}+b^{2}\right)}
\end{array}\right],
$$

where $l$ denotes the pendulum length.

The controllable force $\mathbf{f}_{q}$ acting on the quadrotor with mass $m_{q}$ is defined by the orientation of the quadrotor parameterized with the roll, pitch and yaw Euler angles $\phi, \theta$ and $\psi$ and the collective thrust magnitude $T$ :

$$
\mathbf{f}_{q}=R_{z}(\psi) R_{y}(\theta) R_{x}(\phi)\left[\begin{array}{c}
0 \\
0 \\
T
\end{array}\right]
$$

We denote the mass of the pendulum as $m_{p}=\frac{m_{l}}{2}$ with $m_{l}$ the mass of the load. For now, we do not model any aerodynamic effects, including interference between quadrotor and load. For the following we assume, without loss of generality, that the quadrotor has zero yaw rotation, meaning that the transport frame is aligned with the world coordinate frame.

By the means of the Lagrangian formulation [17] the nonlinear equations of motions (EOM) of the quarotor-pendulum system in the form

$$
\dot{\mathbf{x}}=\mathbf{g}(\mathbf{x}, \mathbf{u})
$$

can be derived, with the state vector $\mathbf{x}$ and the control vector $\mathbf{u}$ defined as

$$
\mathbf{x}=\left[\begin{array}{c}
\mathbf{q} \\
\mathbf{p} \\
\dot{\mathbf{q}} \\
\dot{\mathbf{p}}
\end{array}\right], \mathbf{u}=\left[\begin{array}{c}
\phi \\
\theta \\
T
\end{array}\right]
$$

\section{B. Leader Control Scheme}

We employ a Linear Quadratic Regulator (LQR) to counteract both the deviation of the quadrotors and the pendulums state from the desired state $\mathbf{x}_{\text {des }}$. From the first order Taylor approximation of the EOM (3) we extract the linearized EOM around the desired operating point defined by the desired state $\mathbf{x}_{\text {des }}$ and the corresponding feedforward input $\mathbf{u}_{\text {des }}$ : 


$$
\begin{array}{r}
\dot{\mathbf{x}}-\mathbf{g}\left(\mathbf{x}_{\text {des }}, \mathbf{u}_{\text {des }}\right)=\left.\frac{\delta \mathbf{g}}{\delta \mathbf{x}}\right|_{\mathbf{x}_{\text {des }}, \mathbf{u}_{\text {des }}}\left(\mathbf{x}-\mathbf{x}_{\text {des }}\right) \\
+\left.\frac{\delta \mathbf{g}}{\delta \mathbf{u}}\right|_{\mathbf{x}_{\text {des }}, \mathbf{u}_{\text {des }}}\left(\mathbf{u}-\mathbf{u}_{\text {des }}\right) \\
=A_{c} \tilde{x}+B_{c} \tilde{u}
\end{array}
$$

We use $\tilde{x}$ and $\tilde{u}$ to denote the small deviations from the linearization point. $A_{c}$ and $B_{c}$ are the system matrices for the linearized continuous time system. Based on the corresponding discrete time system $\tilde{x}_{(k+1)}=A_{d} \tilde{x}_{(k)}+B_{d} \tilde{u}_{(k)}$ we compute the infinite horizon LQR feedback gain matrix $K$ that minimizes the cost

$$
J=\sum_{k=0}^{\infty} \tilde{x}_{(k)}^{T} Q \tilde{x}_{(k)}+\tilde{u}_{(k)}^{T} R \tilde{u}_{(k)},
$$

which yields the standard LQR feedback control law $\tilde{u}_{k}=$ $-K \tilde{x}_{k}$. The entries of the positive semi definite penalty matrices $Q$ and $R$ are chosen such that the quadrotor position error $\tilde{\mathbf{q}}$ is punished with penalty weight $k_{q}$, the pendulum position error $\left(\tilde{\mathbf{q}}_{(x, y)}+\tilde{\mathbf{p}}\right)$ with the weight $k_{p}$ and the respective velocity errors with the weights $k_{\dot{q}}$ and $k_{\dot{p}}$. The penalty on the input is split into the attitude penalty $k_{a}$ and the thrust magnitude penalty $k_{T}$. This results in the following structures for $Q$ and $R$ :

$$
\begin{aligned}
Q= & {\left[\begin{array}{cc}
Q_{p, q} & \mathbf{0} \\
\mathbf{0} & Q_{\dot{p}, \dot{q}}
\end{array}\right] } \\
Q_{p, q}= & {\left[\begin{array}{ccccc}
k_{q}+k_{p} & 0 & 0 & k_{p} & 0 \\
0 & k_{q}+k_{p} & 0 & 0 & k_{p} \\
0 & 0 & k_{q} & 0 & 0 \\
k_{p} & 0 & 0 & k_{p} & 0 \\
0 & k_{p} & 0 & 0 & k_{p}
\end{array}\right] } \\
Q_{\dot{p}, \dot{q}}= & {\left[\begin{array}{ccccc}
k_{\dot{q}}+k_{\dot{p}} & 0 & 0 & k_{\dot{p}} & 0 \\
0 & k_{\dot{q}}+k_{\dot{p}} & 0 & 0 & k_{\dot{p}} \\
0 & 0 & k_{\dot{q}} & 0 & 0 \\
k_{\dot{p}} & 0 & 0 & k_{\dot{p}} & 0 \\
0 & k_{\dot{p}} & 0 & 0 & k_{\dot{p}}
\end{array}\right] } \\
R= & {\left[\begin{array}{ccc}
k_{a} & 0 & 0 \\
0 & k_{a} & 0 \\
0 & 0 & k_{T}
\end{array}\right] }
\end{aligned}
$$

Finally the required attitude and thrust is computed as

$$
\mathbf{u}_{(k)}=\mathbf{u}_{d e s}-K\left(\mathbf{x}_{(k)}-\mathbf{x}_{d e s}\right) \text {. }
$$

The above computed command defines a required thrust direction and magnitude that the quadrotor has to assume. We send this command to the quadrotors onboard controller [18], which takes care of controlling the attitude and thrust to the desired values.

In order to take into account the fact that the quadrotor cannot reach the desired attitude instantaneously, we predict the current state using the commands sent in the past and the linearized system derived above. Given the fact that the attitude delay and the thrust delay are of different magnitudes, we use two different prediction horizons for attitude and thrust. Subsequently the new command is computed based on the predicted state.

\section{Follower Control Scheme}

The control scheme of the follower employs the same LQR control technique as described above but applies it only to the plane orthogonal to the transport direction, meaning the parameters $y, z$ and $b$. This ensures a stable heading direction of the transported object. The aim of the follower control scheme in transport direction is to keep the Follower quadrotor exactly above its gripper to minimize the force applied on the load in transport direction. To achieve this we use a PD controller with a force $f_{x}$ in transport direction as output defined as

$$
f_{x}=m_{q} \cdot\left(P_{f} \cdot a+D_{f} \cdot \dot{a}\right),
$$

where $a$ is the offset of the pendulum relative to the quadrotor in transport direction. By adding the force vector resulting form the PD and the LQR controller, the desired attitude and thrust of the Follower is defined and can be sent to the onboard controller.

\section{Trajectory Planning}

Subsequently we describe our trajectory planning approach, that is used in our experiments. For this paper we focus on straight line trajectories at constant height.

As shown in [19] a quadrotor with cable-suspended load is a differentially-flat hybrid (if the cable is not always taut) system with the load position and the quadrotor yaw serving as the flat outputs. This means that by defining the trajectory of the load in time, the state of the quadrotor is defined up to the yaw of the quadrotor, which can be chosen freely. This result can be directly extended to the dual lift scenario. For the following derivation, we assume that the follower quadrotor carries half of the weight of the load with mass $m_{l}$, but does not exert any force on the load in transport direction assumed to be, without loss of generality, the $x$ axis of the world coordinate system.

The force applied to the load at the leader cable connection at position $\mathbf{c}=\left[x_{c}, y_{c}, z_{c}\right]^{T}$ are

$$
\mathbf{f}_{c}=\left[\begin{array}{c}
m_{l} \cdot \ddot{x}_{c} \\
0 \\
g \cdot m_{l} / 2
\end{array}\right]
$$

where $g$ is the gravity constant and $\ddot{x}$ is the trajectory acceleration. Assuming that the cable of length $l$ is completely taut this defines the position if the leader quadrotor as

$$
\mathbf{q}=\mathbf{c}+l \cdot \frac{\mathbf{f}_{c}}{\left\|\mathbf{f}_{c}\right\|}
$$

From the above equation and its derivative the reference states of the leader quadrotor $\mathbf{x}_{r e f}(t)$ during the transport task can be immediately derived.

The required force $\mathbf{f}_{q}$ of the leader quadrotor is defined as the sum of the cable force, the gravitational force and the 
force required to accelerate the quadrotor along the transport trajectory:

$$
\mathbf{f}_{q}=\mathbf{f}_{c}+m_{q} \cdot(\mathbf{g}+\ddot{\mathbf{q}}),
$$

with $\mathbf{g}=[0,0, g]^{T}$. This defines the feed forward input $\mathbf{u}_{\text {ref }}(t)$ along the trajectory. Once the reference states and feed-forward inputs of the leader quadrotor are defined as described above, the control LQR gains of the quadrotor can be defined by linearizing around this points as previously elaborated.

For our experiments we use a piece-wise polynomial trajectory to describe the temporal evolution of the position of the leader-load cable connection point $\mathbf{c}(t)$ from the start to the goal position.

\section{E. Drift and Alignment}

In order for the leader to be able to fully control the position of the payload, as shown in Fig. 2, the follower needs to align its yaw with the leader. If the follower would not do this, the payload would be constrained to move along the axis of the direction of the initial follower yaw, as the control law would preclude the follower from deviating from it. Similarly, the follower needs to track the leader's altitude, as failing to do so could result in the payload obstructing the rotors of either quadrotor. To compensate for misalignment in height and transport direction, the follower tracks the leader at low frequency and adjusts its heading and height using a proportional controller.

\section{EXPERIMENTS}

We validate our method by implementing it on a real system. A lot of effort has been made to run all computations onboard the quadrotors, such that strictly zero communication is required during the object transport (except for evaluation purposes).

\section{A. Hardware}

The hardware used for experiments is depicted in Fig. 4. Each quadrotor is equipped with an Odroid XU4 for general computation and a Pixhawk PX4 for low-level control. The quadrotors attach to the payload using a magnetic gripper, which is equipped with a custom marker for visual tracking. The grippers are tracked with the same camera that is used for visual odometry (VO) and state estimation. This camera is a standard USB camera with a fisheye lens. This choice of lens both maximizes the tracking range for the gripper and makes visual odometry more robust [20]. An April Tag [21] is attached to the back of the leader to simplify the tracking by the follower. Initially, we intended to have the follower track the leader using the same camera that is used for VO. However, we found that the apparent size of the April Tag in the fisheye image is too small to be reliably detected. We therefore equip the follower with a dedicated pinhole camera to track the leader's April Tag.

The payload is a $1 m$ long aluminum rod which weighs $263 \mathrm{~g}$. Using a light payload allows us to perform the experiments with lighter, safer quadrotors - each quadrotor weighs only $800 \mathrm{~g}$. The tether length is $0.4 \mathrm{~m}$, which is an

\begin{tabular}{|l|l|l|l|l|l|l|l|}
\hline$k_{q}$ & $k_{p}$ & $k_{\dot{q}}$ & $k_{\dot{p}}$ & $k_{a}$ & $k_{T}$ & $P_{f}$ & $D_{f}$ \\
\hline 8 & 1 & 2 & 1 & 7 & 0.15 & 25 & 5 \\
\hline
\end{tabular}

TABLE I

GAINS USED IN THE REAL WORLD IMPLEMENTATION.

appropriate length given the room in which the experiments are conducted. The length of the rod has been chosen to ensure that the follower is able to track the leader robustly with the given tracking method. The system is depicted inflight in Fig. 1.

\section{B. Software}

Visual-inertial state estimation is achieved using a pipeline developed in-house [18]. The pipeline uses ROS as middleware, SVO [22] for VO and MSF [23] for fusion of VO and IMU into a visual-inertial odometry (VIO). The tracking of the custom gripper tags is done using a standard Kalman filter with a constant velocity model. It is based on OpenCV and runs in $\sim 10 \mathrm{~ms}$ per frame on the Odroid (1 $\mathrm{ms}$ on an $i 7$ laptop). The follower tracks the April Tag of the leader using an optimized version of the cv2cg April Tag detector [24], which runs in $\sim 100 \mathrm{~ms}$ per frame on the Odroid. While this could allow tracking at $\sim 10 \mathrm{~Hz}$, the frequency is reduced to $5 \mathrm{~Hz}$ in order to free up computational resources.

We use Matlab to derive the equations of motion and to precompute the LQR gains along the trajectory. The gains used in our final system are listed in Table I

For evaluation purposes only, leader, follower and payload are tracked using the motion capture system OptiTrack.

\section{System evaluation}

To evaluate our system, we transport the payload across a linear trajectory of $3.3 \mathrm{~m}$. This allows us to accelerate to and decelerate from $1 \frac{\mathrm{m}}{\mathrm{s}}$ within the motion capture arena at our disposition. At the given accelerations, the quadrotors are outside of near-hover conditions. We use this experiment to validate the stability of our system and to characterize its behavior. We have repeated the experiment until we had at least 10 successful runs; with 13 runs we obtained 11 successful runs, yielding a success rate of $84.6 \%$ after the system has been tuned for the exeriments (good lighting conditions, camera exposures, PID gains, tag detector threshold). All failures that we experienced can be attributed to perception errors (tag detection, tracking loss of SVO at very rapid motions).

The data shown in the results is pre-processed as follows: we are given the state estimate of the leader's VIO, the desired state of the leader expressed in its VIO frame and absolute measurements for the poses of leader, load and follower expressed in the motion capture frame. First, the VIO estimate and desired state are consistently rotated in a way that aligns the desired trajectory with the $\mathrm{x}$-axis. This allows isolating motion parallel to the trajectory from motion lateral to the trajectory. Then, state estimate and desired trajectory are translated such that both their positions at the beginning of trajectory execution coincide with the origin. Finally, the motion capture measurements are all consistently 


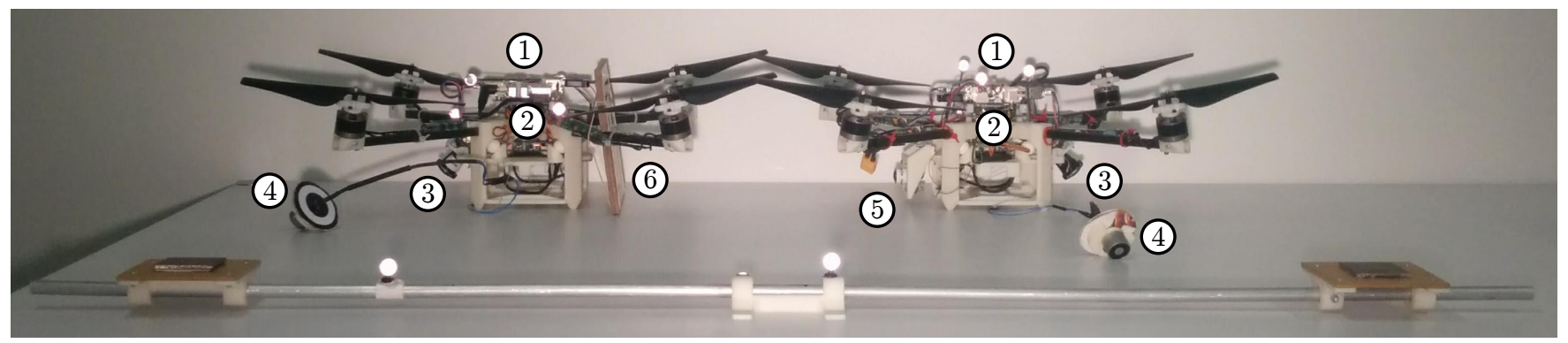

Fig. 4. The hardware used in our experiments: The leader on the left, the follower on the right and the payload on the first plane. Each rigid body is equipped with OptiTrack markers for evaluation only. (1) The computations of each quadrotor are performed on an Odroid XU4. (2) Low-level flight control and IMU is provided by a Pixhawk PX4. (3) 40fps images for flight control and object tracking are obtained from a fisheye camera. (4) Each quadrotor is equipped with a tethered magnetic gripper. A custom visual marker is fixed on top of the gripper. (5) The follower has an extra pinhole camera with which it tracks the April Tag (6) of the leader at $5 \mathrm{~Hz}$.

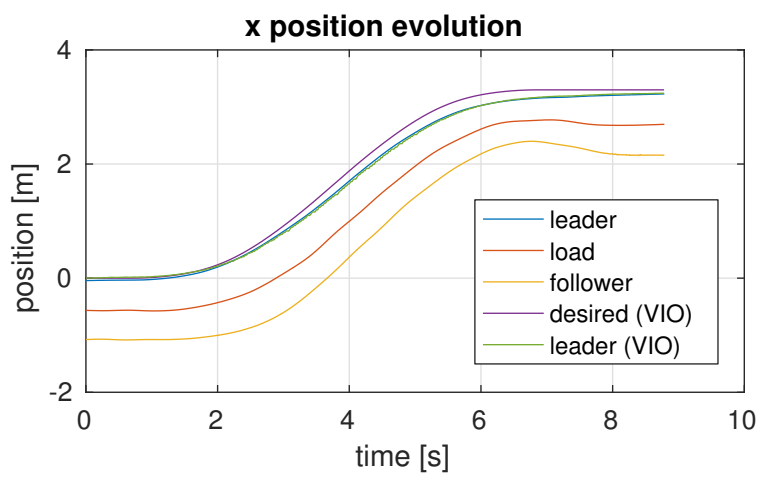

Fig. 5. x-position of the system transporting a payload across $3.3 \mathrm{~m}$, for a single representative experiment. The lines annotated with (VIO) are values originally estimated by the visual-inertial odometry or expressed in its frame, while the other lines represent data measured in motion capture. Note the strong agreement between motion capture and state estimate in this particular experiment.

translated and rotated in yaw only (to preserve the gravity vector) in a way that minimizes the position error between the leader's VIO estimate and its absolute position at all pose estimation times.

\section{Leader tracking evaluation}

An essential part of our system is the follower's ability to track the leader. Should the follower fail to track the leader, the payload would be constrained to motion along the $\mathrm{x}$-axis of the follower. Similarly, inability to track the leader height could lead to a situation where the payload obstructs the rotors of one of the two quadrotors. To validate that in our system the follower is able to avoid such situations, we rerun the first experiment initializing the follower and the load heading in the incorrect direction. Furthermore, we verify the height tracking by observing how the follower adapts to a varying leader altitude. Incidentally, as we experienced height drift in our VIO pipeline during the execution of the experiments, we could observe how the follower is able to track a variyng altitude of the leader.

\section{Results}

Fig. 5 shows the position evolution, parallel to the trajectory, of the system components when transporting our

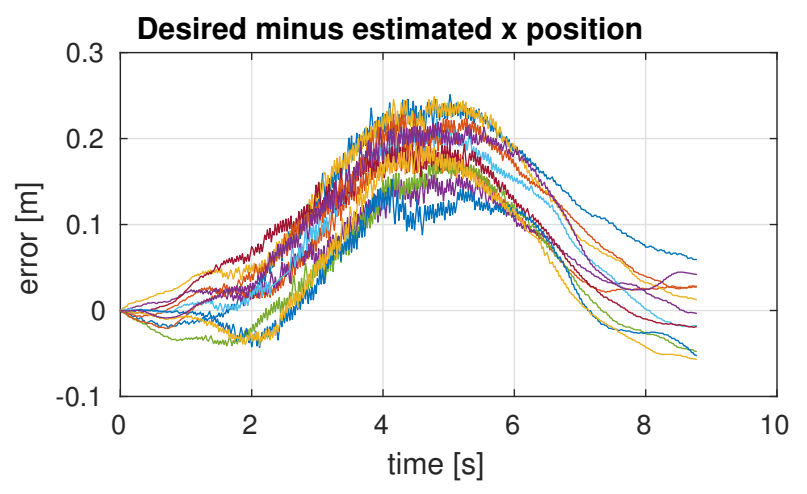

Fig. 6. Error between estimated and desired state of the leader, when transporting a payload across $3.3 \mathrm{~m}$, for the 11 successful runs. Each color denotes a different run.

payload on a straight line across a distance of $3.3 \mathrm{~m}$. This plot shows a single representative instance of the experiment. As we can see, the leader closely follows the planned trajectory at first. However, it starts to diverge from it, only to slowly catch up to it at the end. The divergence between the leader's desired and estimated state for all 11 successful runs is plotted in Fig. 6. As we can see, the divergence reaches a peak of between 10 and $20 \mathrm{~cm}$ at the velocity peak and then slowly makes its way back towards a stable value between $\pm 5 \mathrm{~cm}$. Note the strong jitter in this plot. We attribute it to small errors in pose estimation, which are particularly strong at higher velocities.

In Fig. 5 we further see how load and follower smoothly follow the leader, with some of the follower's inertia apparent in its initial acceleration delay and overshoot as the leader decelerates. Oscillations can only barely be perceived in this plot. The oscillations, particularly of load and follower are more apparent in Fig. 7, which shows the velocity evolution of the system components. The strongest oscillations parallel to the motion occur on load and follower as the acceleration is inverted. Between 3 and 4 seconds, the load velocity overshoots the leader velocity, and both leader and follower start to compensate for this. The follower continues to compensate, until all velocities are roughly matched, at which point the oscillations ebb out. In this plot we can also 


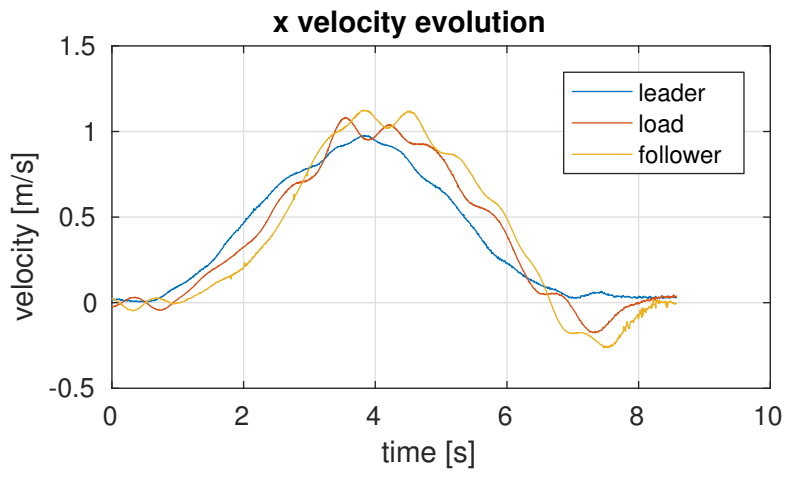

Fig. 7. x-velocity of the system transporting a payload across $3.3 m$, for the same experiment as in Fig. 5.

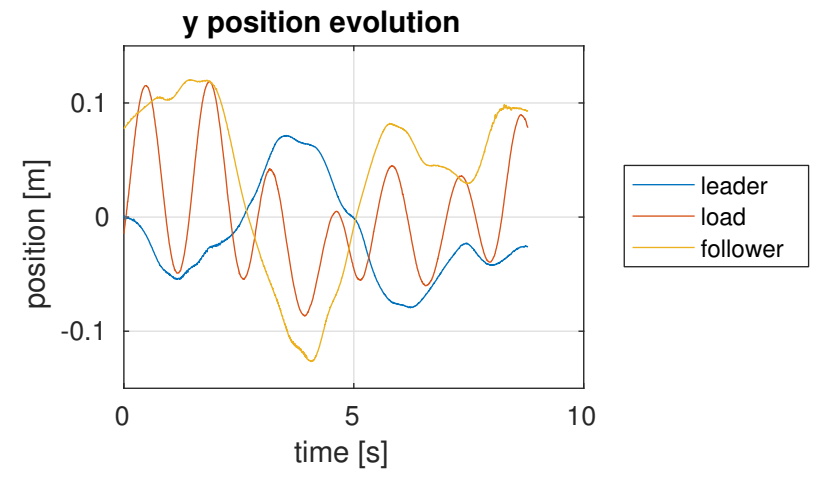

Fig. 8. Lateral positions of the system transporting a payload across $3.3 \mathrm{~m}$, for the same experiment as in Fig. 5.

observe the overshoot of load and follower at the end.

In Fig. 8, deviations and oscillation in y-direction (lateral to the trajectory) are shown for a single run of the experiment. As we can see, the load exhibits some oscillations in spite of our oscillation compensation. Furthermore we can see how leader and follower are deviating from $y=0$ as they are compensating for the load's oscillations. A summary of mean and maximum y error for all runs is provided in table II.

Fig. 9 shows the top-down trajectories of our system if load and follower are initialized with an offset with respect to the transport direction. As we can see, the follower corrects its orientation as soon as the leader moves along the transport direction.

\begin{tabular}{|l|c|c|c|c|}
\hline \multirow{2}{*}{} & \multicolumn{2}{|c|}{ mean [m] } & \multicolumn{2}{c|}{ max [m] } \\
\cline { 2 - 5 } & $\mu$ & $\sigma$ & $\mu$ & $\sigma$ \\
\hline \hline leader & 0.0706 & 0.0316 & 0.1381 & 0.0482 \\
\hline load & 0.0886 & 0.0384 & 0.1957 & 0.0752 \\
\hline follower & 0.1127 & 0.0417 & 0.2187 & 0.0757 \\
\hline
\end{tabular}

TABLE II

ABSOLUTE ERROR WITH RESPECT TO THE TRAJECTORY IN LATERAL

DIRECTION, FOR THE DIFFERENT OBJECTS AND FOR ALL 11

SUCCESSFUL RUNS. FOR EACH RUN, WE ACCUMULATE MEAN AND MAXIMUM ABSOLUTE ERROR, IN METERS, DURING THE RUN. $\mu$ AND $\sigma$ REFER TO MEAN AND STANDARD DEVIATION OF THESE VALUES ACROSS THE EXPERIMENTS.

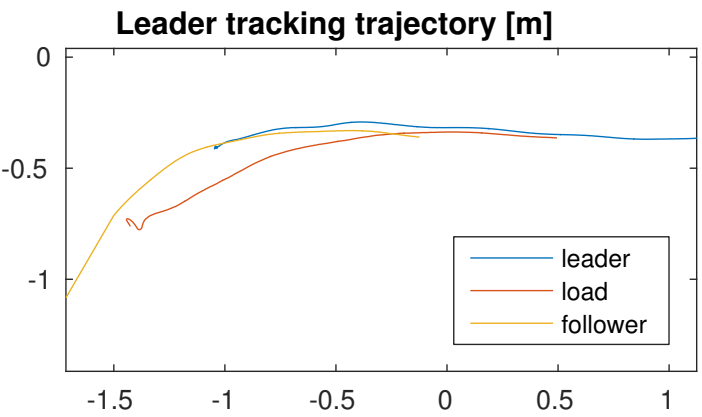

Fig. 9. Leader, load and follower trajectories if load and follower are initialized with an offset in the transport direction. This is the result from an isolated run that is not related to the aforementioned 13 experiment runs.

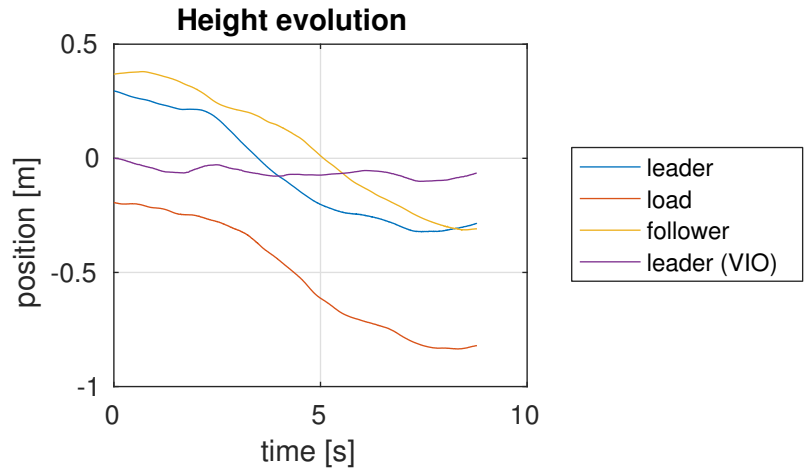

Fig. 10. The follower tracking the leader's height, for the same experiment as in Fig. 5

Finally, Fig. 10 shows that the follower is able to correctly track the leader's height. This plot also shows the aforementioned drift of the VIO height estimate. As we can see, the leader believes that it maintains altitude, while it actually loses altitude. Nevertheless, the follower is able to track its height. This is another advantage of using a system that does not rely on communication for height tracking. The error between leader and follower height for all successful runs is plotted in Fig. 11. While there are some outliers, the general trend is that the difference in height peaks at around $10 \mathrm{~cm}$ during the trajectory execution and returns to a value below $5 \mathrm{~cm}$ afterwards.

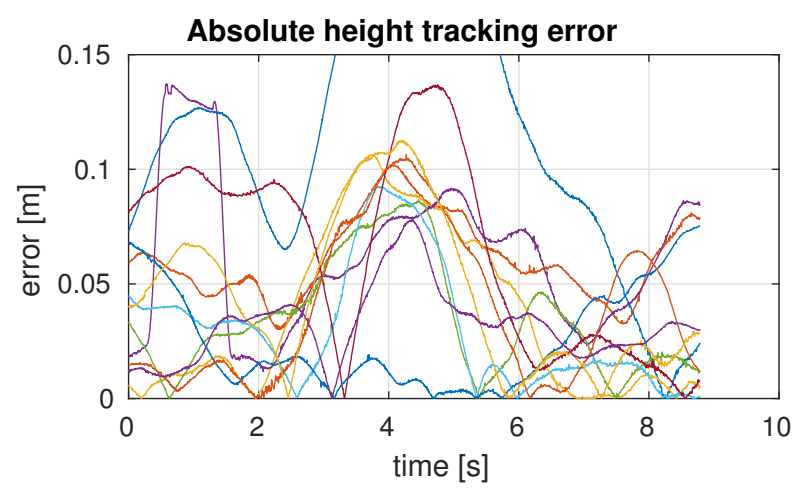

Fig. 11. The absolute height difference between leader and follower, for the 11 successful runs of the experiment. Each color denotes a different run. 


\section{CONCLUSION}

In this paper, we have shown that it is possible for autonomous aerial vehicles to collaboratively transport objects at moderate speeds without explicit communication, using only visual cues. To the best of our knowledge, this is the first collaborative transport system that can cope with trajectories far from quasi-static scenarios. In addition, this is achieved without communication and using only on-board sensing and computation.

\section{REFERENCES}

[1] W. H. Meier and J. R. Olson, "Efficient sizing of a cargo rotorcraft," Journal of Aircraft, 1988.

[2] D. Mellinger and V. Kumar, "Minimum snap trajectory generation and control for quadrotors," in IEEE Int. Conf. on Robotics and Automation, 2011.

[3] L. S. Cicolani and G. Kanning, "General equilibrium characteristics of a dual-lift helicopter system," 1986.

[4] M. Bernard and K. Kondak, "Generic slung load transportation system using small size helicopters," in IEEE Int. Conf. on Robotics and Automation, 2009.

[5] J. Fink, N. Michael, S. Kim, and V. Kumar, "Planning and control for cooperative manipulation and transportation with aerial robots," The Int. Journal of Robotics Research, 2011.

[6] C. De Crousaz, F. Farshidian, and J. Buchli, "Aggressive optimal control for agile flight with a slung load," in IEEE/RSJ Int. Conf. on Intelligent Robots and Systems, 2014.

[7] C. De Crousaz, F. Farshidian, M. Neunert, and J. Buchli, "Unified motion control for dynamic quadrotor maneuvers demonstrated on slung load and rotor failure tasks," in IEEE Int. Conf. on Robotics and Automation, 2015.

[8] S. Tang and V. Kumar, "Mixed integer quadratic program trajectory generation for a quadrotor with a cable-suspended payload," in IEEE Int. Conf. on Robotics and Automation, 2015.

[9] L. S. Cicolani and G. Kanning, "Equations of motion of slung load systems with results for dual lift," 1990.

[10] M. G. Berrios, M. B. Tischler, L. S. Cicolani, and J. Powell, "Stability, control, and simulation of a dual lift system using autonomous r-max helicopters," 70th American Helicopter Society, 2014.

[11] K. Baizid, F. Caccavale, S. Chiaverini, G. Giglio, and F. Pierri, "Safety in coordinated control of multiple unmanned aerial vehicle manipulator systems: Case of obstacle avoidance," in Control and Automation (MED), 2014 22nd Mediterranean Conference of, 2014.

[12] F. Caccavale, G. Giglio, G. Muscio, and F. Pierri, "Cooperative impedance control for multiple uavs with a robotic arm," in IEEE/RSJ Int. Conf. on Intelligent Robots and Systems, 2015.

[13] K. Kosuge and T. Oosumi, "Decentralized control of multiple robots handling an object," in IEEE/RSJ Int. Conf. on Intelligent Robots and Systems, 1996

[14] N. Michael, J. Fink, and V. Kumar, "Cooperative manipulation and transportation with aerial robots," Autonomous Robots, 2011.

[15] M. Manubens, D. Devaurs, L. Ros, and J. Cortés, "Motion planning for 6-d manipulation with aerial towed-cable systems," in Robotics: Science and Systems (RSS), 2013.

[16] T. Sugar and V. Kumar, "Decentralized control of cooperating mobile manipulators," in IEEE Int. Conf. on Robotics and Automation, 1998.

[17] M. Hehn and R. D'Andrea, "A flying inverted pendulum," in IEEE Int. Conf. on Robotics and Automation, 2011.

[18] M. Faessler, F. Fontana, C. Forster, E. Mueggler, M. Pizzoli, and D. Scaramuzza, "Autonomous, vision-based flight and live dense 3d mapping with a quadrotor micro aerial vehicle," Journal of Field Robotics, 2015.

[19] K. Sreenath, T. Lee, and V. Kumar, "Geometric control and differential flatness of a quadrotor uav with a cable-suspended load," in 52nd IEEE Conference on Decision and Control, 2013.

[20] Z. Zhang, H. Rebecq, C. Forster, and D. Scaramuzza, "Benefit of large field-of-view cameras for visual odometry," 2016.

[21] E. Olson, "Apriltag: A robust and flexible visual fiducial system," in IEEE Int. Conf. on Robotics and Automation, 2011.

[22] C. Forster, M. Pizzoli, and D. Scaramuzza, "Svo: Fast semi-direct monocular visual odometry," in 2014 IEEE International Conference on Robotics and Automation (ICRA). IEEE, 2014, pp. 15-22.
[23] S. Lynen, M. W. Achtelik, S. Weiss, M. Chli, and R. Siegwart, "A robust and modular multi-sensor fusion approach applied to mav navigation," in 2013 IEEE/RSJ International Conference on Intelligent Robots and Systems. IEEE, 2013, pp. 3923-3929.

[24] C. Feng and V. R. Kamat, "Plane registration leveraged by global constraints for context-aware aec applications," Computer-Aided Civil and Infrastructure Engineering, 2013. 